PROCEEDINGS OF THE

AMERICAN MATHEMATICAL SOCIETY

Volume 127, Number 11, Pages 3243-3249

S 0002-9939(99)05118-7

Article electronically published on May 4, 1999

\title{
STRONG TYPE ESTIMATE AND CARLESON MEASURES FOR LIPSCHITZ SPACES
}

\author{
ZHIJIAN WU
}

(Communicated by Christopher D. Sogge)

\begin{abstract}
We establish a capacitary strong type estimate for Lipschitz space $\Lambda_{\alpha}^{p, q}$ and characterize the related Carleson measures.
\end{abstract}

\section{$\S 1$. INTRODUCTION}

For $p \geq 1$, let $L^{p}=L^{p}\left(\mathbb{R}^{n}\right)$ and denote its norm by $\|\cdot\|_{p}$. For $p, q \geq 1$ and $0<\alpha<1$, the Lipschitz space $\Lambda_{\alpha}^{p, q}$ consists of all functions $f$ in $L^{p}$ so that

$$
\|f\|_{\Lambda_{\alpha}^{p, q}}=\|f\|_{p}+\left(\int_{\mathbb{R}^{n}} \frac{\|f(\cdot+h)-f(\cdot)\|_{p}^{q}}{|h|^{n+\alpha q}} d h\right)^{1 / q}<\infty .
$$

For any open set $O \subset \mathbb{R}^{n}$, the $\Lambda_{\alpha}^{p, q}$-capacity of $O$ is defined by

$$
\operatorname{cap}_{\Lambda_{\alpha}^{p, q}}(O)=\operatorname{cap}\left(O ; \Lambda_{\alpha}^{p, q}\right)=\inf \left\{\|f\|_{\Lambda_{\alpha}^{p, q}}^{q}: f \geq 1 \text { on } O\right\} .
$$

Let $\mathbb{R}_{+}^{n+1}=\left\{(x, y): x \in \mathbb{R}^{n}, y>0\right\}$ be the upper half space. For any open set $O \subset \mathbb{R}^{n}$, denote by $T(O)$ the tent of $O$ in $\mathbb{R}_{+}^{n+1}$, which is

$T(O)=\left\{(x, y) \in \mathbb{R}_{+}^{n+1}:\right.$ the open ball centered at $x$ with radius $y$ contains in $\left.O\right\}$.

Let $p_{y}(x)=c_{n} \frac{y}{\left(|x|^{2}+y^{2}\right)^{\frac{n+1}{2}}}$ be the Poisson kernel where the constant $c_{n}$ is chosen so that $\int_{\mathbb{R}^{n}} p_{y}(x) d x=1$. For any function $f \in L^{p}$, the harmonic extension of $f$ onto $\mathbb{R}_{+}^{n+1}$ is the convolution between $p_{y}$ and $f$, i.e.

$$
F(x, y)=p_{y} * f(x)=\int_{\mathbb{R}^{n}} p_{y}(x-u) f(u) d u .
$$

The main results of this note are:

Theorem 1. For $q \geq p \geq 1$ and $0<\alpha<1$ there is a constant $C>0$ such that the following strong type estimate

$$
\int_{0}^{\infty} \operatorname{cap}_{\Lambda_{\alpha}^{p, q}}(\{|f|>t\}) d t^{q} \leq C\|f\|_{\Lambda_{\alpha}^{p, q}}^{q}
$$

holds for all $f \in \Lambda_{\alpha}^{p, q}$.

Received by the editors January 25, 1998.

1991 Mathematics Subject Classification. Primary 31C15, 42B25.

Key words and phrases. Lipschitz spaces, capacity, Carleson measures, strong estimate.

The author's research was supported by National Science Foundation DMS 9622890.

(C)1999 American Mathematical Society 
Theorem 2. Suppose $q \geq p>1$ and $0<\alpha<1$. A nonnegative Borel measure $\mu$ on $\mathbb{R}_{+}^{n+1}$ satisfies

$$
\int_{\mathbb{R}_{+}^{n+1}}\left|p_{y} * f(x)\right|^{q} d \mu(x, y) \leq C\|f\|_{\Lambda_{\alpha}^{p, q}}^{q}, \quad \forall f \in \Lambda_{\alpha}^{p, q},
$$

if and only if

$$
\mu(T(O)) \leq C \operatorname{cap}\left(O ; \Lambda_{\alpha}^{p, q}\right), \quad \text { for any bounded open set } O \subset \mathbb{R}^{n} .
$$

Strong type estimates for $L^{p}$ and Sobolev type spaces have been studied systematically in the past. They play an important role in the imbedding theory of function spaces and related areas. We refer the reader to $[\mathrm{A}],[\mathrm{H}],[\mathrm{Z}]$ and $[\mathrm{MS}]$ and the references therein. Estimate (1.2) defines the usual Carleson measure if the space $\Lambda_{\alpha}^{p, q}$ is replaced by $L^{q}$. Therefore, we can say that Theorem 2 gives a characterization for Carleson measures for $\Lambda_{\alpha}^{p, q}$ with $q \geq p>1$ and $0<\alpha<1$. In general, the bounded open set in characterization (1.3) cannot be replaced by an open ball as it can in the characterization for usual Carleson measures. Carleson measures play an important role in harmonic analysis and operator theory on function spaces. For example, it is well-known that the measure $\left|\frac{\partial}{\partial y} p_{y} * f(x)\right|^{2} y d x d y$ is a usual Carleson measure if and only if $f \in$ BMO. Carleson measures for Dirichlet space (analytic) on the unit disk of complex plane is characterized in [S] (see also [KS] for a different characterization). Applications on Hankel operators and related bilinear forms are studied in [RW], [W1] and [W2].

For the sake of simplicity, the letter $C$ always denotes a positive constant which may change from one step to the next. For two positive functions $a$ and $b$, we write $a \asymp b$, if there is a constant $C>0$ such that both $a \leq C b$ and $b \leq C a$ hold.

\section{§2. Proof of Theorem 1}

We start with the smooth truncation. D.R. Adams first used the smooth truncation in $[\mathrm{A}]$ to prove the strong type estimate for Sobolev space on $\mathbb{R}^{n}$. Let $\varphi$ be a nondecreasing function in $C_{0}^{\infty}(\mathbb{R})$ which satisfies

$$
\varphi(t)= \begin{cases}0, & \text { if } t \leq 1 / 2 \\ 1, & \text { if } t \geq 1\end{cases}
$$

Consider the smooth truncation $\left\{F_{j}\right\}_{-\infty}^{\infty}$ :

$$
F_{j}(f)=2^{j} \varphi\left(\frac{|f|}{2^{j}}\right), \quad j=0, \pm 1, \pm 2, \cdots .
$$

The key properties of this smooth truncation are:

$$
\begin{gathered}
0 \leq F_{j}(f) \leq 2^{j} \\
\left\{F_{j}(f)>0\right\} \subset\left\{|f|>2^{j-1}\right\}
\end{gathered}
$$

and if $2^{j-1} \leq|f|<2^{j}$, then

$$
F_{k}(f)= \begin{cases}2^{k}, & \text { for } k<j \\ 0, & \text { for } k>j\end{cases}
$$


Lemma 2.1. For $0<\alpha<1$ and $q \geq p \geq 1$, the smooth truncation $\left\{F_{j}\right\}_{-\infty}^{\infty}$ maps $\Lambda_{\alpha}^{p, q}$ to itself and there is a constant $C>0$ such that

$$
\sum_{j=-\infty}^{\infty}\left\|F_{j}(f)\right\|_{\Lambda_{\alpha}^{p, q}}^{q} \leq C\|f\|_{\Lambda_{\alpha}^{p, q}}^{q}
$$

holds for all $f \in \Lambda_{\alpha}^{p, q}$.

Proof. For any measurable set $E \subset \mathbb{R}^{n}$, denote by $|E|$ the Lebesgue measure of $E$ in $\mathbb{R}^{n}$. It is clear that

$$
\left\|F_{j}(f)\right\|_{p}^{p}=\int_{\left\{F_{j}(f)>0\right\}}\left|F_{j}(f(x))\right|^{p} d x \leq 2^{j p}\left|\left\{|f|>2^{j-1}\right\}\right| .
$$

Since $q / p \geq 1$, we have therefore

$$
\sum_{j=-\infty}^{\infty}\left\|F_{j}(f)\right\|_{p}^{q} \leq \sum_{j=-\infty}^{\infty}\left(2^{j p}\left|\left\{|f|>2^{j-1}\right\}\right|\right)^{q / p} \leq\left(\sum_{j=-\infty}^{\infty} 2^{j p}\left|\left\{|f|>2^{j-1}\right\}\right|\right)^{q / p} .
$$

It is standard that

$$
\int_{\mathbb{R}^{n}}|f(x)|^{p} d x=\int_{0}^{\infty}|\{|f|>t\}| d t^{p} \asymp \sum_{j=-\infty}^{\infty} 2^{j p}\left|\left\{|f|>2^{j}\right\}\right| .
$$

Combining the above two estimates, we obtain

$$
\sum_{j=-\infty}^{\infty}\left\|F_{j}(f)\right\|_{p}^{q} \leq C\left(\int_{0}^{\infty}|\{|f|>t\}| d t^{p}\right)^{q / p}=C\|f\|_{p}^{q}
$$

On the other hand, it is clear that

$$
\begin{aligned}
\sum_{l=-\infty}^{\infty}\left\|F_{l}(f(\cdot+h))-F_{l}(f(\cdot))\right\|_{p}^{q} & \leq\left(\sum_{l=-\infty}^{\infty}\left\|F_{l}(f(\cdot+h))-F_{l}(f(\cdot))\right\|_{p}^{p}\right)^{q / p} \\
& =\left\|\sum_{l=-\infty}^{\infty}\left|F_{l}(f(\cdot+h))-F_{l}(f(\cdot))\right|^{p}\right\|_{1}^{q / p} .
\end{aligned}
$$

Therefore, to complete the proof, it suffices to show that for any $x, h \in \mathbb{R}^{n}$

$$
\sum_{l=-\infty}^{\infty}\left|F_{l}(f(x+h))-F_{l}(f(x))\right|^{p} \leq C|f(x+h)-f(x)|^{p} .
$$

Without loss of generality, assume that $j \geq k$ and

$$
2^{j-1} \leq|f(x+h)|<2^{j} \quad \text { and } \quad 2^{k-1} \leq|f(x)|<2^{k} .
$$

If $j=k$, then

$$
\sum_{l=-\infty}^{\infty}\left|F_{l}(f(x+h))-F_{l}(f(x))\right|^{p}=\left|F_{j}(f(x+h))-F_{j}(f(x))\right|^{p} .
$$

By the mean value theorem, there is a $\xi \in(0,1)$ such that

$$
\begin{aligned}
\left|F_{j}(f(x+h))-F_{j}(f(x))\right|^{p} & =\left|\varphi^{\prime}(\xi)\right|^{p}|| f(x+h)|-| f(x)||^{p} \\
& \leq C|f(x+h)-f(x)|^{p} .
\end{aligned}
$$


If $j \geq k+1$, then

$$
\sum_{l=-\infty}^{\infty}\left|F_{l}(f(x+h))-F_{l}(f(x))\right|^{p}=\left|2^{k}-F_{k}(f(x))\right|^{p}+\left|F_{j}(f(x+h))\right|^{p} .
$$

The two terms on the right hand side above can be estimated, respectively, by

$$
\begin{aligned}
\left|2^{k}-F_{k}(f(x))\right|^{p} & =2^{k p}\left|\varphi(1)-\varphi\left(2^{-k}|f(x)|\right)\right|^{p} \\
& =\left|\varphi^{\prime}(\xi)\right|^{p}\left(2^{k}-|f(x)|\right)^{p} \\
& \leq C|| f(x+h)|-| f(x)||^{p} \\
& \leq C|f(x+h)-f(x)|^{p} ; \\
\left|F_{j}(f(x+h))\right|^{p}= & 2^{j p}\left|\varphi\left(2^{-j}|f(x+h)|\right)-\varphi(1 / 2)\right|^{p} \\
& =\left|\varphi^{\prime}(\eta)\right|^{p}\left(|f(x+h)|-2^{j-1}\right)^{p} \\
\leq & C|f(x+h)-f(x)|^{p} .
\end{aligned}
$$

The proof is complete.

We now prove Theorem 1. It is standard that

$$
\int_{0}^{\infty} \operatorname{cap}_{\Lambda_{\alpha}^{p, q}}(\{|f|>t\}) d t^{q} \asymp \sum_{k=-\infty}^{\infty} 2^{k q} \operatorname{cap}_{\Lambda_{\alpha}^{p, q}}\left(\left\{|f|>2^{k}\right\}\right) .
$$

Since $2^{-k} F_{k}(f) \geq 1$ on the set $\left\{|f|>2^{k}\right\}$, we have

$$
\operatorname{cap}_{\Lambda_{\alpha}^{p, q}}\left(\left\{|f|>2^{k}\right\}\right) \leq 2^{-k q}\left\|F_{k}(f)\right\|_{\Lambda_{\alpha}^{p, q}}^{q} .
$$

Therefore,

$$
\sum_{k=-\infty}^{\infty} 2^{k q} \operatorname{cap}_{\Lambda_{\alpha}^{p, q}}\left(\left\{|f|>2^{k}\right\}\right) \leq \sum_{k=-\infty}^{\infty}\left\|F_{k}(f)\right\|_{\Lambda_{\alpha}^{p, q}}^{q} \leq C\|f\|_{\Lambda_{\alpha}^{p, q}}^{q} .
$$

This last inequality is obtained by using Lemma 2.1 .

Consider the homogeneous space $\dot{\Lambda}_{\alpha}^{p, q}$, which is the completion of $C_{0}^{\infty}\left(\mathbb{R}^{n}\right)$ under the norm

$$
\|f\|_{\dot{\Lambda}_{\alpha}^{p, q}}=\left(\int_{\mathbb{R}^{n}} \frac{\|f(\cdot+h)-f(\cdot)\|_{p}^{q}}{|h|^{n+\alpha q}} d h\right)^{1 / q}
$$

The relation between $\Lambda_{\alpha}^{p, q}$ and $\dot{\Lambda}_{\alpha}^{p, q}$ is $\Lambda_{\alpha}^{p, q}=\dot{\Lambda}_{\alpha}^{p, q} \cap L^{p}$. One can define $\dot{\Lambda}_{\alpha}^{p, q}$-capacity similarly. The above discussion proves also the following result.

Theorem $\mathbf{1}^{\prime}$. For $q \geq p \geq 1$ and $0<\alpha<1$ there is a constant $C>0$ such that the following strong type estimate

$$
\int_{0}^{\infty} \operatorname{cap}_{\dot{\Lambda}_{\alpha}^{p, q}}(\{|f|>t\}) d t^{q} \leq C\|f\|_{\dot{\Lambda}_{\alpha}^{p, q}}^{q}
$$

holds for all $f \in \dot{\Lambda}_{\alpha}^{p, q}$.

Denote by $B(x ; r)$ the open ball in $\mathbb{R}^{n}$ centered at $x$ with radius $r>0$. We gather the estimates of $\dot{\Lambda}_{\alpha}^{p, q}$-capacity and $\Lambda_{\alpha}^{p, q}$-capacity for balls in the following lemma. 
Lemma 2.2. Let $B_{r}=B(x ; r)$. Then

(a) $\operatorname{cap}\left(B_{r} ; \dot{\Lambda}_{\alpha}^{p, q}\right) \asymp\left|B_{r}\right|^{\left(\frac{1}{p}-\alpha\right) q}$;

(b) $\operatorname{cap}\left(B_{r} ; \Lambda_{\alpha}^{p, q}\right) \asymp \max \left(\left|B_{r}\right|^{\frac{q}{p}},\left|B_{r}\right|^{\left(\frac{1}{p}-\alpha\right) q}\right)$.

Proof. Let $f_{r}(x)=f\left(\frac{x}{r}\right)$. It is easy to verify that

$$
\left\|f_{r}\right\|_{p}=r^{\frac{n}{p}}\|f\|_{p} \quad \text { and } \quad\left\|f_{r}\right\|_{\dot{\Lambda}_{\alpha}^{p, q}}=r^{\frac{n}{p}-\alpha}\|f\|_{\dot{\Lambda}_{\alpha}^{p, q}} .
$$

These imply the desired results.

\section{§3. Proof of Theorem 2}

Recall that $B(x ; r)$ denotes the open ball in $\mathbb{R}^{n}$ centered at $x$ with radius $r>0$. For a function $f$ defined on $\mathbb{R}^{n}$, the Hardy-Littlewood maximum function $M(f)$ is defined by

$$
M(f)(x)=\sup _{r>0} \frac{1}{|B(x ; r)|} \int_{B(x ; r)}|f(u)| d u .
$$

The proof of next lemma is due to M. Taibleson.

Lemma 3.1. For $0<\alpha<1$ and $p>1$, the Hardy-Littlewood maximum function operator is bounded on $\Lambda_{\alpha}^{p, q}$. More precisely, there is a constant $C>0$ such that

$$
\|M(f)\|_{\Lambda_{\alpha}^{p, q}} \leq C\|f\|_{\Lambda_{\alpha}^{p, q}}
$$

hold for all $f \in \Lambda_{\alpha}^{p, q}$.

Proof. It is well-known that $M$ is bounded on $L^{p}$, i.e. $\|M(f)\|_{p} \leq C\|f\|_{p}$. For $h \in \mathbb{R}^{n}$, let $T_{h}$ be the translation by $h$, i.e. $T_{h} f(x)=f(x+h)$. By definition (1.1), it suffices to show that

$$
\left\|T_{h} M(f)-M(f)\right\|_{p} \leq C\left\|T_{h} f-f\right\|_{p} .
$$

It is easy to see that $T_{h}$ commutes with $M$, i.e. $T_{h} M(f)=M\left(T_{h} f\right)$. Hence we have

$$
\left|T_{h} M(f)-M(f)\right|=\left|M\left(T_{h} f\right)-M(f)\right| \leq M\left(\left|T_{h} f-f\right|\right) .
$$

Taking $L^{p}$ norm on both ends of the above inequality, by the boundedness of $M$ on $L^{p}$, we obtain the desired result.

For $x \in \mathbb{R}^{n}$ denote by $\Gamma(x)=\left\{(u, t) \in \mathbb{R}_{+}^{n+1}:|u-x|<t\right\}$, the cone with vertex at $x$. For a function $g$ defined on $\mathbb{R}_{+}^{n+1}$, the nontangential maximal function of $g$ is a function on $\mathbb{R}^{n}$ defined by

$$
N(g)(x)=\sup _{(u, t) \in \Gamma(x)}|g(u, y)| .
$$

Lemma 3.2. Let $\mu$ be a nonnegative Borel measure on $\mathbb{R}_{+}^{n+1}$. Then for any measurable function $g$ on $\mathbb{R}_{+}^{n+1}$ and any $t>0$

$$
\mu(\{|g(x, y)|>t\}) \leq \mu(T(\{N(g)(x)>t\})) .
$$

Proof. Denote by $\Omega=\left\{(x, y) \in \mathbb{R}_{+}^{n+1}:|g(x, y)|>t\right\}$. Associating each point $(x, y) \in \Omega$ with the ball $B(x ; y)$ and the tent

$$
T(B(x ; y)),
$$

clearly, $(x, y) \in T(B(x ; y))$, and therefore tents $\{T(B(x ; y)):(x, y) \in \Omega\}$ cover $\Omega$. 
For any compact subset $K$ of $\Omega$, by compactness, there are finitely many tents $\left\{T\left(B_{k}\right)\right\}_{1}^{m}$ in (3.1) cover $K$. For any $u \in B_{k}=B(x ; y)$, we have $|u-x|<y$, or equivalently $(x, y) \in \Gamma(u)$, and hence we know that $N(g)(u) \geq|g(x, y)|>t$. This implies that

$$
\bigcup_{k=1}^{m} B_{k} \subset\{N(g)>t\}
$$

Hence, by the fact that $\bigcup_{k=1}^{m} T\left(B_{k}\right)=T\left(\bigcup_{k=1}^{m} B_{k}\right)$, we obtain

$$
K \subset T\left(\bigcup_{k=1}^{m} B_{k}\right) \subset T(\{N(g)>t\}) .
$$

This is enough to conclude the desired result.

We now prove Theorem 2. The approach in the following has its root in $[\mathrm{S}]$; there Stegenga proved a result for Carleson measures for the Dirichlet space (analytic) on the unit disk of the complex plane which is similar to the case $p=q=2$ here.

We prove the "only if" part of Theorem 2 first. Given a bounded open set $O \subset \mathbb{R}^{n}$, by the definition of $\Lambda_{\alpha}^{p, q}$-capacity, there is a function $f \in \Lambda_{\alpha}^{p, q}$ such that $f \geq 1$ on the set $O$ and $\|f\|_{\Lambda_{\alpha}^{p, q}}^{q} \leq 2 \operatorname{cap}\left(O ; \Lambda_{\alpha}^{p, q}\right)$. We can assume further that $f \geq 0$ on $\mathbb{R}^{n}$, because it is easy to show (similar to the proof of Lemma 3.1) that $\||f|\|_{\Lambda_{\alpha}^{p, q}} \leq\|f\|_{\Lambda_{\alpha}^{p, q}}$. Since $(x, y) \in T(O)$ is equivalent to $B(x ; y) \subset O$, we have for any $(x, y) \in T(O)$,

$$
\begin{aligned}
p_{y} * f(x) & =\int_{\mathbb{R}^{n}} p_{y}(x-u) f(u) d y \\
& \geq \int_{O} p_{y}(x-u) d u \\
& \geq \int_{B(x ; y)} p_{y}(x-u) d u \\
& =\int_{|u|<1} p_{1}(u) d u \\
& =\delta
\end{aligned}
$$

Therefore, we obtain

$$
\mu(T(O)) \leq \delta^{-q} \int_{\mathbb{R}_{+}^{n+1}}\left|p_{y} * f(x)\right|^{q} d \mu(x, y) \leq C\|f\|_{\Lambda_{\alpha}^{p, q}}^{q} \leq C \operatorname{cap}\left(O ; \Lambda_{\alpha}^{p, q}\right) .
$$

To prove the "if" part of Theorem 2, we consider the measure

$$
\mu_{k}=\left.\mu\right|_{T(B(0 ; k))}, \quad k>0 .
$$

For any $f \in \Lambda_{\alpha}^{p, q}$, let $F(x, y)=p_{y} * f(x)$. By Lemma 3.2 and the standard result that $N(F)(x) \leq C M(f)(x)$, we have

$$
\begin{aligned}
\mu_{k}(\{|F(x, y)|>t\}) & \leq \mu_{k}(T(\{N(F)(x)>t\})) \\
& \leq \mu_{k}(T(\{M(f)>t / C\})) .
\end{aligned}
$$

It is clear that for any pair of open sets $O_{1}, O_{2} \subset \mathbb{R}^{n}$, we have $T\left(O_{1} \cap O_{2}\right)=$ $T\left(O_{1}\right) \cap T\left(O_{2}\right)$, and if $O \subset O^{*}$ then $\operatorname{cap}_{\Lambda_{\alpha}^{p, q}}(O) \leq \operatorname{cap}_{\Lambda_{\alpha}^{p, q}}\left(O^{*}\right)$. We can continue 
the above estimate by

$$
\begin{aligned}
& =\mu(T(\{M(f)>t / C\} \cap B(0 ; k))) \\
& \leq \operatorname{cap}_{\Lambda_{\alpha}^{p, q}}(\{M(f)>t / C\} \cap B(0 ; k)) \\
& \leq \operatorname{cap}_{\Lambda_{\alpha}^{p, q}}(\{M(f)>t / C\}) .
\end{aligned}
$$

Hence, by Theorem 1 and Lemma 3.1, we obtain

$$
\begin{aligned}
\int_{T(B(0 ; k))}\left|p_{y} * f(x)\right|^{q} d \mu(x, y) & =\int_{0}^{\infty} \mu_{k}\left(\left\{\left|p_{y} * f(x)\right|>t\right\}\right) d t^{q} \\
& \leq \int_{0}^{\infty} \operatorname{cap}_{\Lambda_{\alpha}^{p, q}}(\{M(f)>t / C\}) d t^{q} \\
& \leq C\|M(f)\|_{\Lambda_{\alpha}^{p, q}}^{q} \\
& \leq C\|f\|_{\Lambda_{\alpha}^{p, q}}^{q} .
\end{aligned}
$$

Finally, letting $k$ go to infinity, we get the desired inequality.

Similarly, using Theorem 1', one can get the following result.

Theorem 2'. Suppose $q \geq p>1$ and $0<\alpha<1$. A nonnegative Borel measure $\mu$ on $\mathbb{R}_{+}^{n+1}$ satisfies

$$
\int_{\mathbb{R}_{+}^{n+1}}\left|p_{y} * f(x)\right|^{q} d \mu(x, y) \leq C\|f\|_{\Lambda_{\alpha}^{p, q}}^{q}, \quad \forall f \in \dot{\Lambda}_{\alpha}^{p, q},
$$

if and only if

$$
\mu(T(O)) \leq C \operatorname{cap}\left(O ; \dot{\Lambda}_{\alpha}^{p, q}\right), \quad \text { for any bounded open set } O \subset \mathbb{R}^{n} .
$$

\section{REFERENCES}

[A] D. R. Adams, On the existence of capacitary strong type estimates in $\mathbb{R}^{n}$, Arkiv Math 14 (1976), 125-140. MR 54:5822

[H] K. Hansson, Imbedding theorems of Sobolev type in potential theory, Math. Scand. 45 (1979), 77-102. MR 81j:31007

[KS] R. Kerman and E. Sawyer, Carleson measures and multipliers of Dirichlet spaces, Trans. Amer. Math. Soc. 309 (1988), 87-98. MR 89i:30044

[MS] V. G. Maźya and T. O. Shaposhnikova, Theory of Multipliers in Spaces of Differentiable Functions, Pitman Advanced Publishing Program, Boston and London. MR 87j:46074

[RW] R. Rochberg and Z. Wu, A new characterization of Dirichlet type spaces and its applications, Illinois J. Math. (1) 37 (1993), 101-122. MR 93j:30039

[S] D. Stegenga, Multipliers of the Dirichlet space, Illinois J. Math. 24 (1980), 113-139. MR 81a:30027

[St] E. M. Stein, Singular Integrals and Differentiability Properties of Functions, Princeton Univ. Press, Princeton Univ., 1970. MR 44:7280

[W1] Z. Wu, The predual and second predual of $W_{\alpha}$, J. Funct. Anal. (2) 116 (1993), 314-334. MR 94g:46033

[W2] _ A class of bilinear forms on Dirichlet type spaces, J. London Math. Soc. (2) 54 (1996), 498-514. MR 97h:46039

[W3] Clifford analysis and commutators on the Besov spaces, J. Funct. Anal., to appear.

[Z] W. P. Ziemer, Weakly Differentiable Functions, Springer-Verlag, New York, 1989. MR 91e: 46046

Department of Mathematics, University of Alabama, Tuscaloosa, Alabama 35487

E-mail address: zwu@euler.math.ua.edu 